\title{
Cryo-FIB Workflow for Fabricating Thin Lamellae Using TESCAN S8000G
}

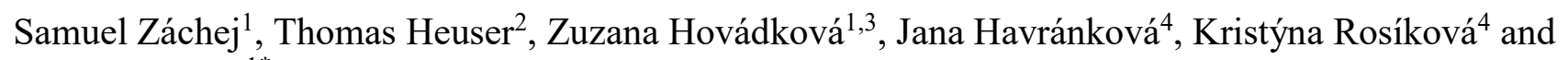
Rostislav Váňa ${ }^{1 *}$

1. TESCAN Brno, Brno, Czech Republic.

2. Vienna Biocenter Core Facility, Vienna, Austria.

3. Brno University of Technology, Brno, Czech Republic.

4. TESCAN ORSAY HOLDING, Brno, Czech Republic.

* Corresponding author: rostislav.vana@tescan.com

Understanding cellular ultrastructure and function, ideally in the native state requires a powerful analytical method. Cryo-electron tomography (cryo-ET) has become a well-established tool to study frozen vitrified specimens in a close-to-native state. A large proportion of samples in structural biology, such as plungefrozen eukaryotic cells, exceed the accessible dimensions $(\sim 500 \mathrm{~nm})$ for TEM imaging and are highly topographic. Cryo-sectioning is the standard method used for thinning and slicing such samples. However, data quality is easily degraded due to artefacts caused by knife marks, compression or crevasses. [1]

Focused ion beam (FIB) nanofabrication has been employed in material sciences to prepare ultra-thin 3D structures from bulk material for transmission electron microscopy (TEM). The same technique can be applied to biological samples. A few attempts have succeeded in lift-out of thin lamellae followed by attachment to a half-moon TEM grid [2]. This promising method involves many delicate steps, and can be successfully used for extracting a specific slice from a bulk sample. Another approach is to specifically thin down a plunge-frozen specimen directly on the TEM grid. The sample stays fixed to the TEM grid, which provides mechanical support and thermal protection during transfer. Furthermore, both FIB methods can be navigated by fluorescence microscopy to unveil functional data from a specific region of interest.

We used the latter technique for preparing Escherichia coli and Saccharomyces cerevisiae cells for cryoET. The sample was applied to golden TEM grids and plunge-frozen in liquid ethane using a Leica GP2. The sample was then loaded to a pre-tilted sample holder and transferred using a Leica VCT500 to the TESCAN S8000G FIB-SEM [3], equipped with a cryo-stage and cryo-shield. Non-conductive cells can be a challenge when faced with an unknown sample, but we used prior knowledge of the shape and dimensions of our sample and coated the cells with organometallic platinum $(10 \mathrm{~nm})$ before the transfer.

Prior to FIB milling, the deposition of a protective layer is needed. Rough surface can cause unequal sputtering of the subsurface material, create a curtaining effect. Moreover, FIB-induced deposition using a gas precursor is limited, as the precursor gas freezes immediately upon contact with the cold surface. We tuned the protocol [4] and prepared a reproducible preset for reliable deposition at cryogenic temperature using FIB curing of the selected area. Then, the FIB was used to open parallel windows into the sample and create the lamellae. The orientation of the grid in the pre-tilted holder is almost parallel to the ORAGE ${ }^{\mathrm{TM}}$ Ga FIB column. Geometry of the FIB milling was designed to speed up the process by using high milling currents and to protect the sample from heat damage at the same time. A large portion of the sample was removed with high current ion beam $(>500 \mathrm{pA})$, forming a wedge, which was later thinned using small currents to avoid FIB-induced-heat damage of the sample. Final polishing was done using low current ion beam, resulting in thin $(<300) \mathrm{nm}$ lamellae. The deposition and milling procedures 
were designed and semi-automated in the scanning generator DrawBeam ${ }^{\mathrm{TM}}$. The TEM grid containing the lamella was transferred to the cryo-TEM and a tilt series was collected at $200 \mathrm{keV}$ beam energy. The acquisition was controlled using SerialEM software.

\section{References:}

[1] A Al-Amoudi, D Studer and J Dubochet, Journal of Structural Biology 150 (2005), p. 109.

[2] S Rubino et al., Journal of Visualized Experiments 89 (2014).

[3] P Sytar, J Jiruse and A Zavodny, Microscopy and Microanalysis 23(S1) (2017), p. 38.

[4] M.F.Hayles et al., Journal of Microscopy 226 (2007), p. 263.

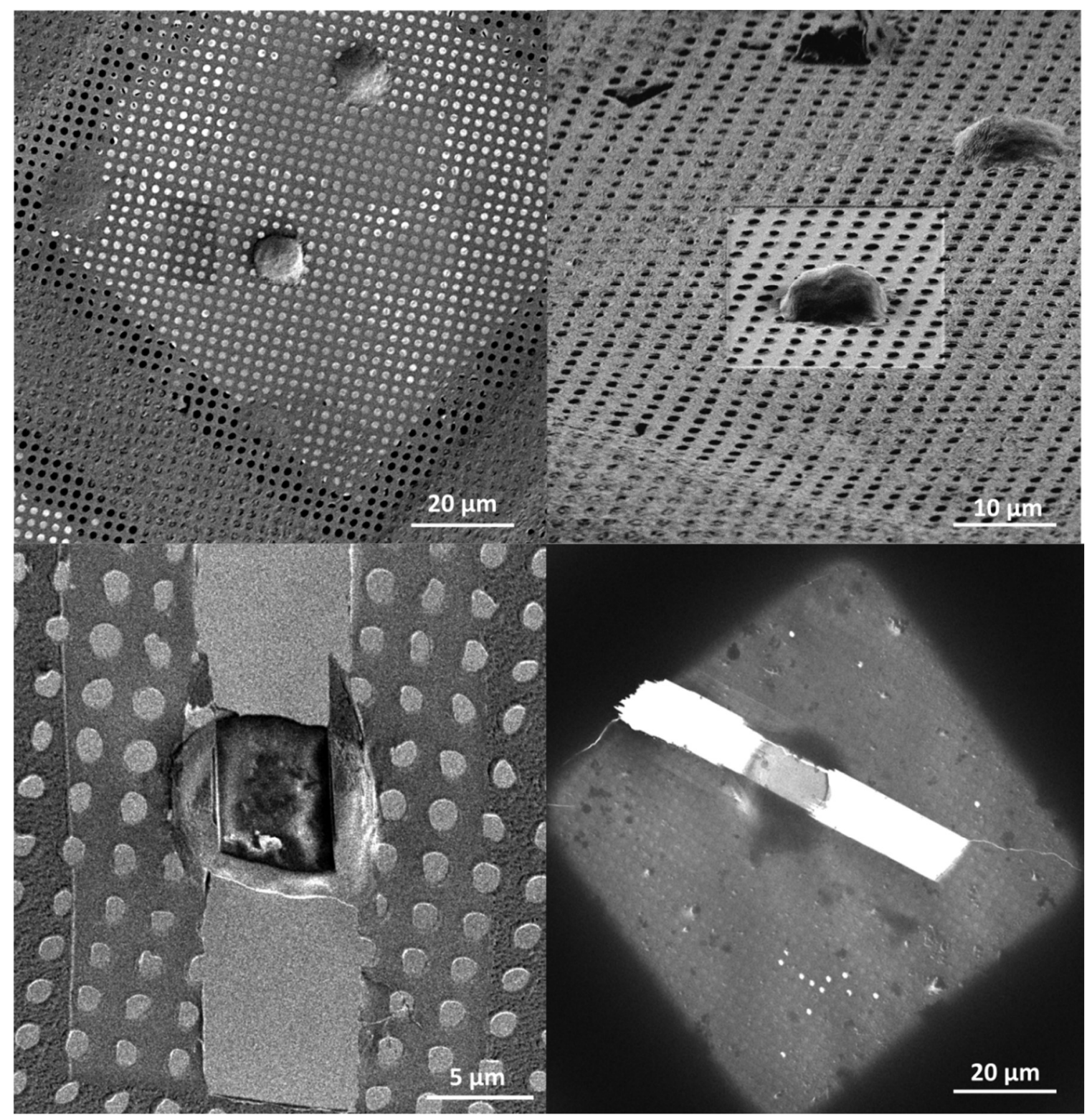

Figure 1. FIB workflow for lamella preparation of eukaryotic cells. Top left - Selected region on the plunge-frozen sample; Top right - FIB view after GIS deposition; Bottom left - lamella before final polishing (thickness approx. $1 \mu \mathrm{m})$; Bottom right - overview of the lamella in the cryo-TEM $(200 \mathrm{keV}$, field of view $100 \mu \mathrm{m})$. 